\title{
Single impact trauma in human early-stage osteoarthritic cartilage: Implication of prostaglandin D2 but no additive effect of IL-1 $\beta$ on cell survival
}

\author{
HELGA JOOS $^{1 *}$, CATHRIN HOGREFE $^{1 *}$, LUCIA RIEGER $^{1}$, LUTZ DÜRSELEN $^{2}$, \\ ANITA IGNATIUS $^{2}$ and ROLF E. BRENNER ${ }^{1}$ \\ ${ }^{1}$ Division for Biochemistry of Joint and Connective Tissue Diseases, Department of Orthopedics; \\ ${ }^{2}$ Institute of Orthopedic Research and Biomechanics, University of Ulm, Ulm, Germany
}

Received January 24, 2011; Accepted March 8, 2011

DOI: $10.3892 / \mathrm{ijmm} .2011 .694$

\begin{abstract}
Injury to articular cartilage is often associated with an inflammatory reaction and frequently results in the development of post-traumatic osteoarthritis (post-traumatic OA). Cell death, inflammation and loss of proteoglycans participate in these mechanisms with p38MAPK being one of the pivotal signaling kinases. Therefore, the interaction of trauma and of the pro-inflammatory cytokine IL-1 $\beta$ was investigated in an in vitro tissue model of human osteoarthritic cartilage. Trauma was induced by impacting cartilage explants with a drop-tower system and its effect was measured in terms of cell survival, gene expression and the release of mediators. In addition, the effect of concomitant IL-1 $\beta$ stimulation and p38MAPK inhibition by SB203580 was investigated. We found a significant decrease in chondrocyte viability after trauma, but no additional effect of IL-1 $\beta$ stimulation. SB203580 had a tendency to improve cell survival suggesting a role for p38 signaling in cell viability after impact in an inflammatory environment. We showed that various mediators are released in response to trauma with or without IL-1 $\beta$ stimulation, differing in composition and time response. Trauma resulted in an increased release of IL-6, whereas TNF- $\alpha$ and IL-1 $\beta$ release was unaffected. Prostaglandin (PG) and NO synthesis pathways were both affected by trauma and/ or IL-1 $\beta$. We demonstrate for the first time an elevated release of prostaglandin D2 (PGD2) by human articular cartilage in response to a single mechanical impact. The up-regulation of mediators was time-dependent, with a more early increase
\end{abstract}

Correspondence to: Professor Rolf E. Brenner, Division for Biochemistry of Joint and Connective Tissue Diseases, Department of Orthopedics, University of Ulm, Oberer Eselsberg 45, D-89081 Ulm, Germany

E-mail: rolf.brenner@uni-ulm.de

*Contributed equally

Key words: cartilage, trauma, IL-1 $\beta$, p38MAPK, cell death, prostaglandin D2, prostaglandin E2 of PGD2 compared to prostaglandin E2 (PGE2) and a late induction of NO by co-stimulation with IL-1 $\beta$ between 6 and $24 \mathrm{~h}$.

\section{Introduction}

Traumatic joint damage is a known risk factor for the subsequent development of post-traumatic osteoarthritis (post-traumatic OA) and is most commonly accompanied by macroscopic lesions of the articular surface (1). Impact loads at energies insufficient to grossly disrupt the articular surface can result in post-traumatic OA as well (2). Frequently, joint traumas also include an injury of the adjacent soft tissues like the synovium or ligaments which lead to a significant increase of the pro-inflammatory cytokines, IL- $1 \beta$ and TNF- $\alpha$, in the synovial fluid with a peak of cytokine levels within $24 \mathrm{~h}$ of trauma (3). Based on these findings, a concerted action of trauma-induced and cytokine-induced processes in cartilage is expected to be responsible for the development of posttraumatic OA.

The underlying mechanisms are complex and only partially understood. The substantial increase in chondrocyte death after joint trauma is believed to play a pivotal role in post-traumatic OA pathogenesis. Mechanical stress generates necrosis (4) and induces apoptosis (5) involving the release of reactive oxygen species (ROS) (6). Furthermore, an impact results in the early loss of glycosaminoglycans due to mechanical disruption of the cartilage extracellular matrix and a subsequent induction of degrading enzymes supporting cartilage degradation $(7,8)$.

The increase of pro-inflammatory cytokines after joint injury (3) also supports the progression of post-traumatic OA. Mediators like IL-1 $\beta$ are potent activators of cartilage degradation by decreasing matrix production and increasing the production of catabolic enzymes and inflammatory mediators $(9,10)$. Interactions of traumagenic and inflammation-derived effects are described. Mechanical injury potentiates the catabolic effects of TNF- $\alpha$ and IL- $1 \alpha$ causing an increased degradation of proteoglycans in cartilage $(11,12)$.

New therapeutic approaches try to address the underlying pathogenetic mechanisms in post-traumatic OA in order to surpass the effectiveness of surgical restoration and of the 
removal of intraarticular blood. Efforts to reduce chondrocyte death or dysfunction after joint injury may be a useful strategy to delay or prevent the onset of post-traumatic OA (2). For example, treatment of injured cartilage with the membrane-stabilizing surfactant P188 has been shown to prevent chondrocytes from undergoing early necrosis (4) as well as subsequent apoptosis in adjacent regions (13). A reduction of trauma-induced apoptosis in vitro has also been observed after treatment with caspase inhibitors or substances that reduce the amount of $\operatorname{ROS}(5,6,14,15)$.

In addition to the prevention of chondrocyte death by ROS diminishment, the reduction of inflammatory and degradative processes in cartilage seems to attenuate the development of post-traumatic OA, while the inhibition of cyclooxygenase could lower the trauma-induced release of prostaglandin E2 (PGE2) and could reduce apoptosis (16). The inhibition of matrix metalloproteinases was shown to reduce the loss of glycosaminoglycans following in vitro cartilage injury (7).

These findings implicate the involvement of many different processes in the initiation of post-traumatic OA. We, therefore, hypothesized that inhibition of a central signaling pathway mediating trauma-induced and inflammation-induced effects could positively affect several processes and thus be an advantageous therapeutic approach. One of the relevant signaling pathways is that of MAPK (mitogen-activated protein kinase), especially the p38MAPK pathway, as it plays a pivotal role in the inflammatory processes in joint destruction. Inhibition of the p38MAPK by the inhibitor SB203580 was previously shown to reduce PGE2 and NO-release of chondrocytes in an in vitro inflammation model (17) and demonstrated an attenuation of cartilage degradation in vivo (18). Furthermore, p38MAPK is thought to be a key player in mechanical stressinduced signaling as activation of p38MAPK by mechanical stress was observed in bovine cartilage and human chondrocytes $(19,20)$. Inhibition of p38 activation after cartilage injury is also responsible for the chondroprotective effects of the P188 surfactant (13).

We, therefore, investigated the interaction of trauma and of pro-inflammatory cytokines in an in vitro tissue model of human osteoarthritic cartilage concerning cell survival, gene expression and release of mediators. Inhibition with the p38MAPK inhibitor, SB203580, was investigated as a possible therapeutic approach.

\section{Materials and methods}

Cartilage explants. Human osteoarthritic cartilage was obtained from donors undergoing total knee joint replacement due to OA. Informed consent was obtained from all the patients according to the terms of the Ethics Committee of the University of Ulm. Overall, tissue samples from 32 patients were included in the study. The mean age of the donors was 66 years (range 53-82 years). Only tissue regions of the femoral condyles were used that had a macroscopically smooth surface and no severe osteoarthritic changes.

Full-thickness cartilage explants, $6 \mathrm{~mm}$ in diameter, were harvested by punching the cartilage with a biopsy punch and separating the cartilage from the underlying subchondral bone with a scalpel. Each explant was weighed and cultivated in complete medium consisting of 1:1 DMEM/Ham's F12

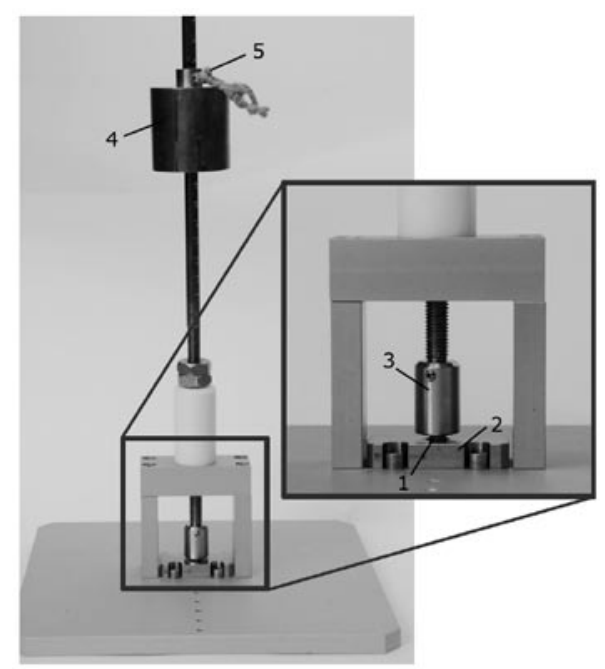

Figure 1. Drop-tower for impaction of cartilage explants. The indentor (3) was placed on the cartilage explant (1), lying on a loading platen (2). The dropping mass (4) was held in place by a height-adjustable cotter pin (5). By manual release of the cotter pin the mass fell down and transferred the drop energy to the indentor.

supplemented with $10 \%$ fetal bovine serum, $0.5 \%$ penicillin/ streptomycin (PAA Laboratories, Pasching, Austria), 0.5\% L-glutamine and $10 \mu \mathrm{g} / \mathrm{ml}$ 2-phospho-L-ascorbic acid trisodium salt (Sigma-Aldrich, Fluka, Seelze, Germany) for $24 \mathrm{~h}$ in an incubator $\left(37^{\circ} \mathrm{C}, 21 \% \mathrm{O}_{2}, 95 \%\right.$ humidity). Afterwards, the explants were cultivated up to 15 days in serum-free medium consisting of DMEM supplemented with $1 \%$ sodium pyruvate, $0.5 \%$ L-glutamine, $1 \%$ non-essential amino acids, $0.5 \%$ penicillin/streptomycin and $0.1 \%$ serum effective substitute (SES-1 Solution A). SES-1 Solution B was freshly added to the medium in each case. All chemicals were purchased from Biochrom (Berlin, Germany) unless specified otherwise.

Impact loading. A specially designed drop-tower (Fig. 1) was used to impart loads to an indenter, a flat-faced steel rod of $15 \mathrm{~mm}$ in diameter, resting on the explant surface. Two levels of impact energy were applied by dropping a $600 \mathrm{~g}$ mass from a height of $10 \mathrm{~cm}$ and $20 \mathrm{~cm}$ on the indenter, which resulted in an impact energy of $0.59 \mathrm{~J}$ and $1.18 \mathrm{~J}$, respectively. The dropping mass, held in place by a height-adjustable cotter pin, that was released manually, was removed from the indenter immediately after impact. Unloaded explants served as controls.

In addition to the impact loading, some of the cartilage explants were also treated with IL-1 $\beta(10 \mathrm{ng} / \mathrm{ml})$ (PeproTech, Hamburg, Germany) and/or with SB203580 $(1 \mu \mathrm{M})$ (gift of Ratiopharm, Ulm, Germany). Twenty-four hours after the described treatments the cartilage explants and the culture media were harvested.

mRNA isolation and cDNA synthesis. For total RNA isolation the cartilage explants were frozen in liquid $\mathrm{N}_{2}$. After pulverization with a microdismembrator (Sartorius BBI Systems, Melsungen, Germany), RNA was isolated using the Lipid Tissue Mini kit (Qiagen, Hilden, Germany). RNA was reverse transcribed with the Omniscript RT kit (Qiagen) and used for 
A

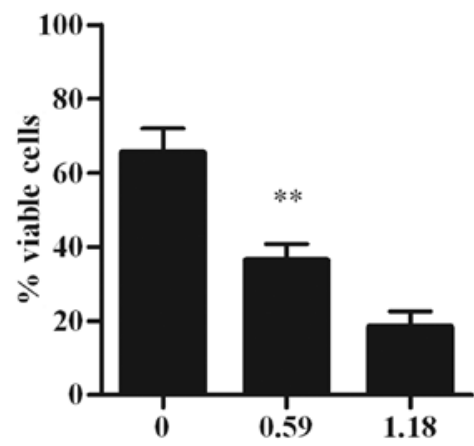

Trauma $[\mathbf{J}]$
B

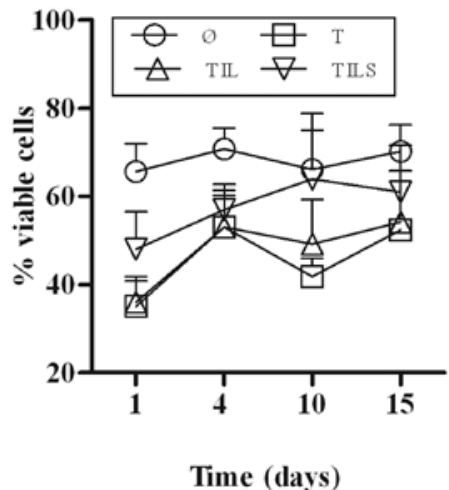

$\mathbf{C}$

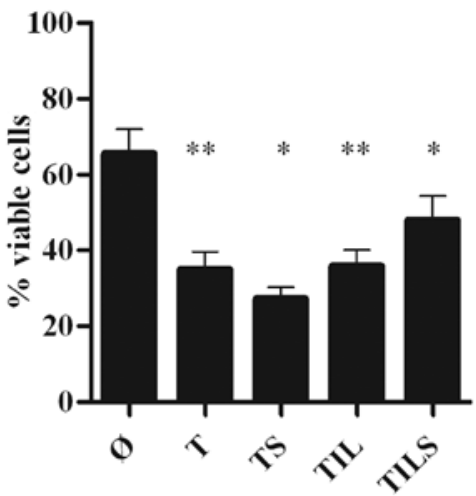

Figure 2. Chondrocyte viability in response to trauma, IL-1 $\beta$ stimulation and p38MAPK inhibition. (A) Cartilage explants were traumatized with $0.59 \mathrm{~J}(\mathrm{n}=11)$ or $1.18 \mathrm{~J}(\mathrm{n}=2)$. (B and C) Cartilage explants were traumatized ( $0.59 \mathrm{~J})$ and stimulated or not with IL-1 $\beta(10 \mathrm{ng} / \mathrm{ml})$. SB203580 $(1 \mu \mathrm{M})$ was used for p38MAPK

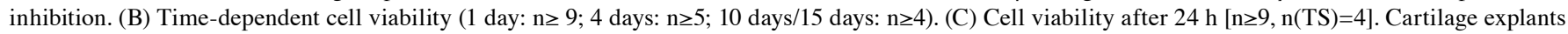
without treatments served as control. The values represent the mean \pm SEM. ${ }^{*} \mathrm{p}<0.05,{ }^{* *} \mathrm{p}<0.01$. $\varnothing$, control; T, trauma; IL, IL-1 $\beta$; S, SB203580.

quantitative real-time PCR-analysis (ABIPrism 7000 system; Applied Biosystems, Darmstadt, Germany).

Real-time polymerase chain reaction (RT-PCR). Relative gene expression analysis $\left(2^{-\Delta \Delta \mathrm{Ct}}\right.$ method) using the Platinum ${ }^{\circledR}$ $\mathrm{SYBR}^{\circledR}$ qPCR SuperMix UDG (Invitrogen, Darmstadt, Germany) was used for NOS2A, 5'-ATTCACTCAGCTGTG CATCG-3' (forward) and 5'-TCAGGTGGGATTTCGAAG AG-3' (reverse); COX2, 5'-CCTTGGGTGTCAAAGGTAA-3' (forward) and 5'-GGCAAAGAATGCAAACATCA-3' (reverse); PTGES, 5'-CCCCCAGTATTGCAGGAG-3' (forward) and 5'-GGAAGACCAGGAAGTGCATC-3' (reverse). The TaqMan ${ }^{\circledR}$ Gene Expression Master mix (Applied Biosystems) was used for the TaqMan Gene Expression assay (Applied Biosystems) Hs00168748_m1 (PTGDS). The Power SYBR-Green PCR Master mix (Applied Biosystems) was used for 18SrRNA, 5'-CGCAGCTAGGAATAATGGAATAGG-3' (forward) and 5'-CATGGCCTCAGTTCCGAAA-3' (reverse), which served as the endogenous control.

ELISA and NO assays. Absolute concentrations of nitrite, a stable end-product of the NO metabolism, were determined in the media of the tissue culture using the Griess assay (Griess Reagent System; Promega, Mannheim, Germany) according to the manufacturer's instructions. PGE2 production was measured in the media by the PGE2 ELISA Correlate EIA ${ }^{\mathrm{TM}}$ kit (Biotrend, Assay Designs, Cologne, Germany).

PGD2 production was measured with the prostaglandin D2 EIA kit (Cayman, Biomol, Hamburg, Germany) and IL-6, IL-1 $\beta$ and TNF- $\alpha$ production was measured by the human Quantikine ELISA kits for IL-6, IL-1 $\beta /$ IL-1F2 and TNF- $\alpha$, respectively (all R\&D Systems, Wiesbaden, Germany), according to the manufacturer's instructions.

Cytotoxicity assay. A Live/Dead ${ }^{\circledR}$ viability/cytotoxity assay (Molecular Probes, Invitrogen) was performed to determine the percentage of viable cells, which was carried out by staining the chondrocytes of an unfixed tissue section $(0.5 \mathrm{~mm}$ thickness) with $1 \mu \mathrm{M}$ Calcein $\mathrm{AM}$ and $2 \mu \mathrm{M}$ ethidium homodimer-1 for $30 \mathrm{~min}$. After washing in PBS, the tissue sections were microscopically analyzed with the help of a z-stack module (AxioVision software; Carl Zeiss, Jena, Germany).

Statistical analysis. For standardization of the gene expression levels determined by real-time PCR-analysis, the mRNA expression was normalized to that of 18SrRNA. Differential regulation was determined by calculating the ratios of gene expression under different treatments. The data represent the means \pm SEM. A two-tailed paired t-test was used to evaluate significant differences in the means.

\section{Results}

In vitro trauma model. To establish a reliable in vitro trauma model, different energies were used for cartilage impact and the percentage of vital cells was subsequently determined (Fig. 2A). To preserve enough vital cells, the lower impact energy of $0.59 \mathrm{~J}$ was chosen for further experiments.

Detection of pro-inflammatory cytokines. Using ELISAs, we determined the release of IL-1 $\beta$, IL- 6 and TNF- $\alpha$ after trauma and the release of IL- 6 and TNF- $\alpha$ after trauma plus IL-1 $\beta$ stimulation by the cartilage explants of at least 4 donors. Fig. 3A and C show significant elevations of IL-6 and TNF- $\alpha$ release. IL-1 $\beta$ release was not affected by trauma (data not shown). Control stimulation of unimpacted cartilage explants with IL-1 $\beta(n \geq 4)$ revealed 6 -fold and 15 -fold increases in IL- 6 and TNF- $\alpha$ release, respectively (data not shown). Furthermore, we could detect a time-dependent release of IL-6 (Fig. 3B).

Influence of trauma, pro-inflammatory stimulation and additional p38MAPK inhibition on cell viability. Impacted explants were cultivated with or without IL-1 $\beta$ for up to 15 days and processed for Live/Dead staining (Fig. 2B). Quantitative analysis revealed a reduction of vital cells from 65 to $35 \% 24 \mathrm{~h}$ after trauma but no further enhancement of trauma-induced cell death by IL-1 $\beta$ within 15 days of cultivation post-trauma. As a significant effect of trauma on cell viability was already observed after $24 \mathrm{~h}$, this time point was chosen for further analyses of gene expression and mediator release. 
A

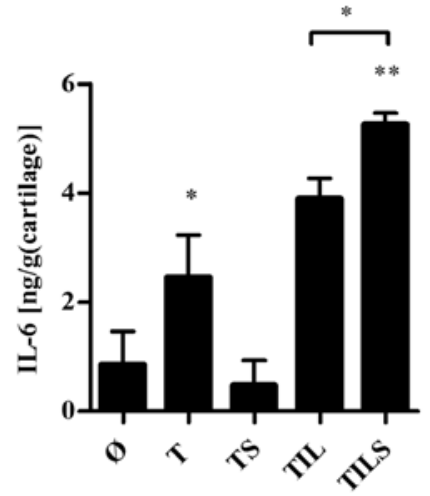

B

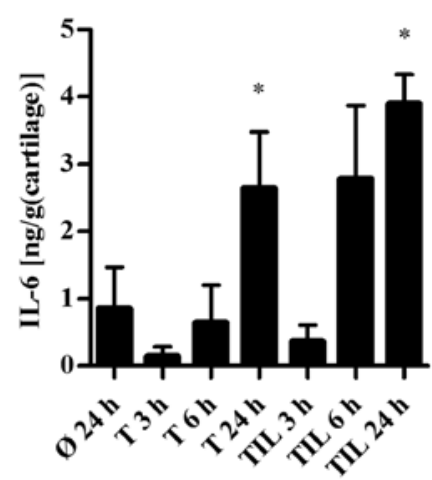

C

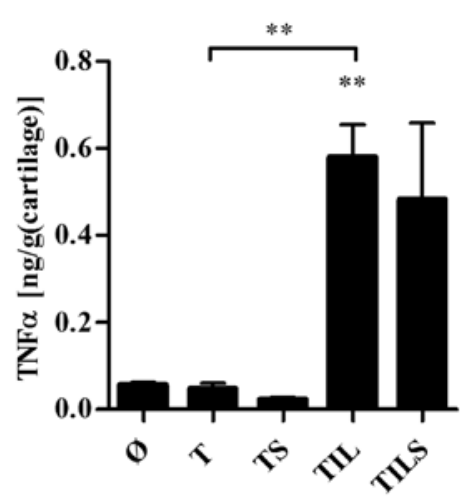

Figure 3. Effects of trauma, IL-1 $\beta$ stimulation and p38MAPK inhibition on cytokine release. Cartilage explants were traumatized (0.59 J) and stimulated or not with IL-1 $\beta(10 \mathrm{ng} / \mathrm{ml})$. SB203580 $(1 \mu \mathrm{M})$ was used for p38MAPK inhibition. Cartilage explants without treatments served as control. (A) Release of IL-6 after $24 \mathrm{~h}(\mathrm{n} \geq 8, \mathrm{n}(\mathrm{TS})=4)$. (B) Time-dependent release of IL-6 $(\mathrm{n} \geq 3)$ and (C) release of TNF- $\alpha$ after $24 \mathrm{~h}(\mathrm{n} \geq 3)$. The values represent the mean \pm SEM. ${ }^{*} \mathrm{p}<0.05,{ }^{* *} \mathrm{p}<0.01 . \varnothing$, control; T, trauma; IL, IL-1 $\beta ; \mathrm{S}, \mathrm{SB} 203580$.

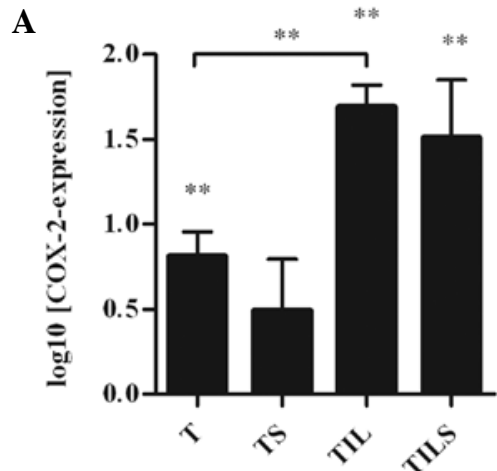

D

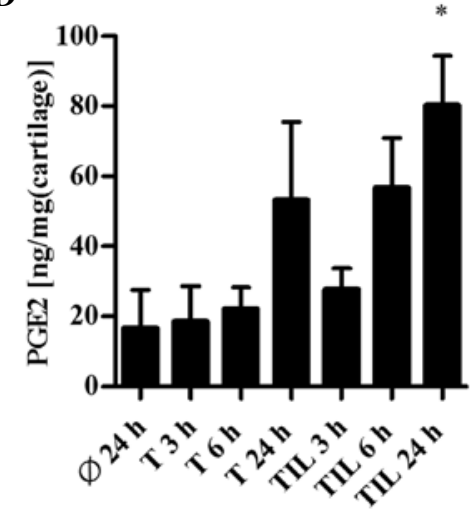

B

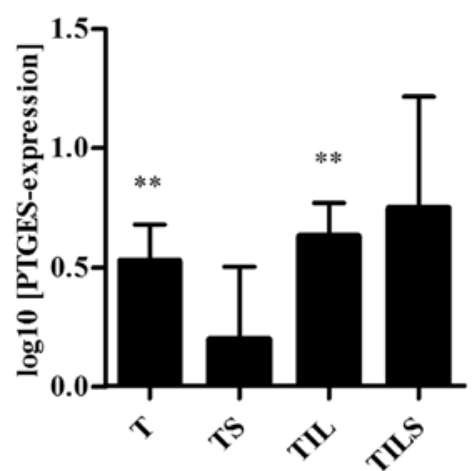

$\mathbf{E}$

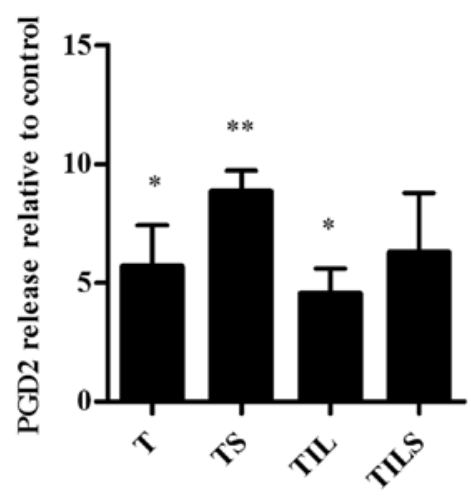

C

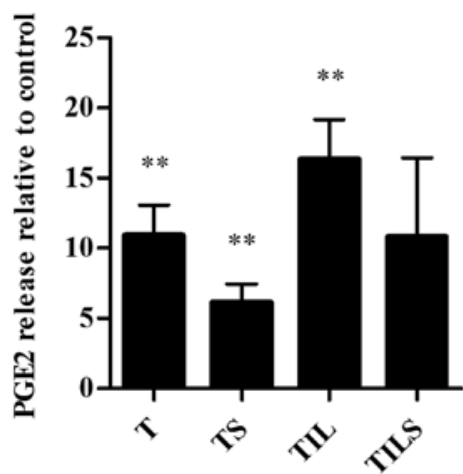

$\mathbf{F}$

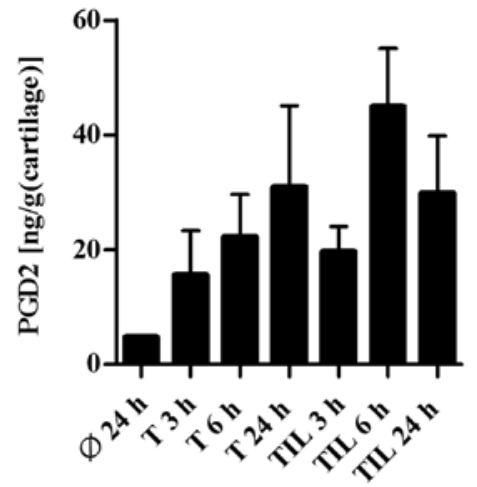

Figure 4. Effects of trauma, IL-1 $\beta$ stimulation and p38MAPK inhibition on the prostaglandin synthesis pathway. Cartilage explants were traumatized (0.59 J) and stimulated or not with IL-1 $\beta(10 \mathrm{ng} / \mathrm{ml})$. SB203580 $(1 \mu \mathrm{M})$ was used for p38MAPK inhibition. Cartilage explants without treatments served as control. mRNA-expression of (A) COX2 [ $\mathrm{n} \geq 13, \mathrm{n}(\mathrm{TS})=5, \mathrm{n}(\mathrm{TILS})=7]$ and (B) PTGES [ $\mathrm{n} \geq 10, \mathrm{n}(\mathrm{TS} / \mathrm{TILS})=3]$ after $24 \mathrm{~h}$ was determined by real-time PCR. Expression levels are normalized to 18SrRNA and shown relative to control. (C) Release of PGE2 relative to control after $24 \mathrm{~h}$ (n $\geq 7$ ). (D) Time-dependent release of PGE2 ( $n=5)$. (E) Release of PGD2 relative to control after $24 \mathrm{~h}(\mathrm{n} \geq 5)$. (F) Time-dependent release of PGD2 ( $\mathrm{n}=3$ ). The values represent the mean \pm SEM. ${ }^{*} \mathrm{p}<0.05,{ }^{* *} \mathrm{p}<0.01$. Ø, control; T, trauma; IL, IL-1 $\beta ;$ S, SB203580.

To address the question for the involvement of the p38MAPK signaling pathway in this context, we investigated the effect of SB203580 on cell viability in impacted cartilage explants with or without IL-1 $\beta$ stimulation after $24 \mathrm{~h}$ (Fig. 2C). Though not significant, SB203580 could increase the mean number of viable cells from $36 \%$ to $48 \%$ in impacted, stimulated explants. Neither IL-1 $\beta$ stimulation nor treatment with SB203580 alone influenced cell viability, as determined in a control experiment (data not shown).
Influence of trauma, pro-inflammatory stimulation and p38MAPK inhibition on cell metabolism. Quantitative analysis of the prostaglandin synthesis pathway revealed significant effects of trauma as well as trauma combined with IL-1 $\beta$ stimulation, whereas p38MAPK inhibition acted differentially, as shown in Fig. 4. The release of PGE2 correlated with the gene expression of the corresponding enzymes, COX2 and PTGES (Fig. 4A-C). The production of PGD2 was elevated by the different treatments (Fig. 4E) whereas the 
A

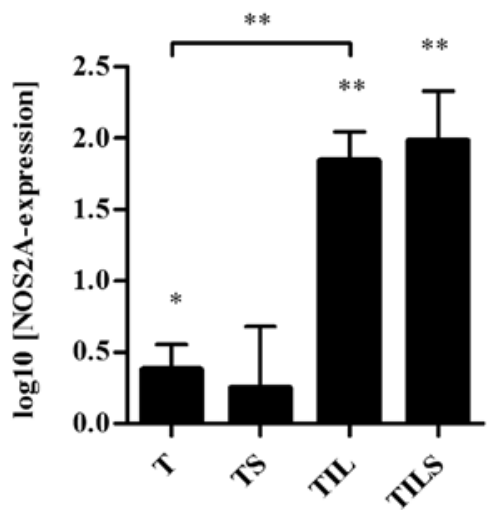

B

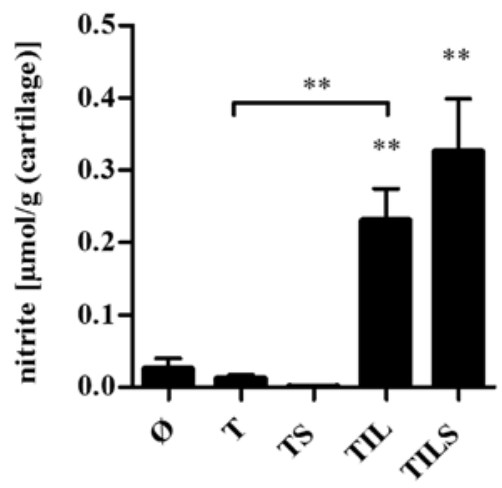

C

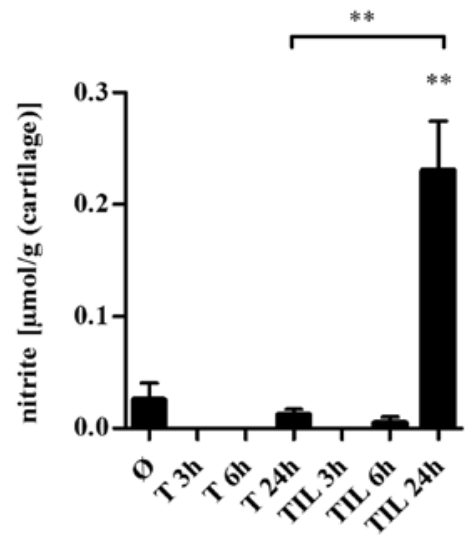

Figure 5. Effects of trauma, IL-1 $\beta$ stimulation and p38MAPK inhibition on NO synthesis pathway. Cartilage explants were traumatized (0.59 J) and stimulated or not with IL-1 $\beta(10 \mathrm{ng} / \mathrm{ml})$. SB203580 $(1 \mu \mathrm{M})$ was used for p38MAPK inhibition. Cartilage explants without treatments served as a control. (A) mRNA-expression of NOS2A after $24 \mathrm{~h}[\mathrm{n} \geq 14, \mathrm{n}(\mathrm{TS})=5, \mathrm{n}(\mathrm{TILS})=7]$ was determined by real-time PCR. Expression levels were normalized to 18SrRNA and shown relative to control. (B) Release of nitrite after $24 \mathrm{~h}(\mathrm{n} \geq 8)$. (C) Time-dependent release of nitrite [ $\mathrm{n} \geq 8, \mathrm{n}(\mathrm{T} 3 \mathrm{~h} / 6 \mathrm{~h}, \mathrm{TIL} 3 \mathrm{~h} / 6 \mathrm{~h})=3$ ]. The values represent the mean \pm SEM. ${ }^{*} \mathrm{p}<0.05,{ }^{* *} \mathrm{p}<0.01$. $\varnothing$, control; T, trauma; IL, IL-1 $\beta$; S, SB203580.

mRNA expression level of PTGDS was not significantly altered (data not shown). In a control experiment ( $\mathrm{n} \geq 2)$, IL-1 $\beta$ stimulation of unimpacted explants caused a 112-fold and 22-fold up-regulation of COX2 and PTGES gene expression, accompanied by a 16-fold and 5-fold increase in PGE2 and PGD2 release, respectively (data not shown). The effect of the impact combined with the IL-1 $\beta$ stimulation on PGE2 synthesis exceeded the trauma effect, whereas both treatments had a comparable effect on PGD2 release. COX2 induction was significantly higher after concomitant trauma and IL-1 $\beta$ stimulation compared to trauma alone, but in the same range as COX2-induction by IL-1 $\beta$ alone. Ultimately, IL-1 $\beta$ amplified the trauma-induced effect on COX2 gene expression and PGE2 release. The time course of PGE2 and PGD2 release is shown in Fig. 4D and F, indicating different time responses of the two prostaglandins.

The effects of impact, impact combined with IL-1 $\beta$ stimulation and additional p38MAPK inhibition on the NO synthesis pathway are shown in Fig5. IL-1 $\beta$ stimulation of unimpacted explants $(n=9)$ revealed a 220 -fold up-regulation of NOS2A and a 10-fold increase in NO production (data not shown). The time course of NO release (Fig. 5C) revealed a late induction compared to the prostaglandins.

To address the trauma and inflammatory effects on further mediators involved in cell death, the release of Fas ligand (FasL) was investigated (data not shown). No detectable release of FasL was found by any of the analyzed cartilage explants.

\section{Discussion}

This study investigated the combined effects of cartilage trauma and IL-1 $\beta$ stimulation as well as the involvement of the p38MAPK signaling pathway on the cell viability in human osteoarthritic cartilage explants. Twenty-four hours post trauma, a significant reduction (30\%) of viable cells was observed, which is comparable to other human trauma models $(16,21)$. An additional stimulation with IL-1 $\beta$ did not enhance cell death in our tissue culture model. Though IL-1 was described to synergistically enhance proteoglycan degradation in mechanically injured cartilage (11), we could find no additive effect of IL-1 $\beta$ on cell loss. In order to elucidate the involved mediators and their relation to the observed chondrocyte death, we investigated the synthesis pathways of PGE2, PGD2 and NO as well as the secretion of IL-6, TNF- $\alpha$, IL-1 $\beta$ and FasL. In summary, an induction of PGD2, PGE2 and IL- 6 could be observed by trauma $24 \mathrm{~h}$ post-impact whereas concomitant IL-1 $\beta$ stimulation led to elevated levels of PGE2, IL-6, NO, TNF- $\alpha$ and PGD2, with levels of the latter subsiding by $24 \mathrm{~h}$. Keeping in mind the reduced amount of viable cells after trauma, there is an additive effect detectable in the release of IL-6 and PGE2 after trauma in combination with IL-1 $\beta$ stimulation compared to an individual treatment by impact or IL-1 $\beta$ stimulation, respectively.

An impact-induced IL-6 up-regulation in healthy human ankle cartilage has been described by Bajaj et al (13), indicating an unequivocal trauma-effect in articular cartilage with and without slight pre-existing damage and implicating IL-6 as a possible mediator of cell death. The additive IL-6 induction by IL-1 $\beta$ in impacted explants in our study, however, caused no additional cell loss. Sui et al described an amplifying effect of IL-6 in a bovine cartilage injury model concerning proteoglycan catabolism (12). But IL-6 seems not to be responsible for cell death, which was also described for another cartilage injury model (22).

In accordance to another study (16), the analysis of the PGE2 synthesis pathway in impacted explants showed a significant up-regulation of COX2 and PTGES gene expression as well as of PGE2 release $24 \mathrm{~h}$ post-trauma, accompanied by an increase in cell death. Cartilage injury with concomitant IL-1 $\beta$ stimulation resulted in a significantly stronger COX2induction and tended to increase the PGE2 release compared to trauma alone. This elevation was not reflected in an enhanced cell death, indicating a secondary role of PGE2 in cell death signaling, as previously described (23).

For the first time, we could describe an increased PGD2 release after single mechanical impact of human articular cartilage. This finding correlates to effects observed in sheared chondrocytes in cell culture (23), indicating a general effect of mechanical stimulation in chondrocytes which is preserved in the context of cartilage matrix. Trauma as well as trauma plus 
IL-1 $\beta$ stimulation resulted in a comparable PGD2 induction which is in agreement with the observed comparable levels of cell death under both conditions, supporting a major role of PGD2 in chondrocyte death (23). In contrast to the elevated PGD2 level, PTGDS gene expression was not altered $24 \mathrm{~h}$ post-impact with or without IL-1 $\beta$ stimulation, which could be explained by an early peak of PTGDS-expression which was fading away by $24 \mathrm{~h}$. Though we found no additional IL-1 $\beta$-mediated PGD2 induction, Zayed et al described IL-1 $\beta$ to induce PTGDS-expression in cell culture after 12-24 h (24). These findings indicate that trauma may be responsible for the early PGD2-induction in our model and that traumainduced PGD 2 possibly prevents a subsequent IL-1 $\beta$-induced up-regulation of PTGDS gene expression, to some extent by a negative feedback mechanism (24).

There was no significant induction of NO release by trauma, though NOS2A gene expression was slightly elevated after impact. Fermor et al, however, showed that specific conditions of compression increase NOS2A expression and NO production in porcine cartilage explants (25). The discrepancies between their study and our results could be due to differences in the conditions of mechanical stimulation or in the species used. Furthermore, the level of NO production in osteoarthritic cartilage could be elevated per se resulting in a lower effect of additional mechanical stress, as the NO-induction by OA-relevant cytokines like IL-1 $\beta$ or TNF- $\alpha$ was found to be substantially stronger than that by mechanical stress (25). The strong IL-1 $\beta$-effect on NOS2A gene expression and NO production in cartilage indicates that most of the NO release is probably induced by inflammatory processes of the injured tissue (cartilage and adjacent tissue) rather than by primary mechanical stress. Although NO has been reported to be a key inducer of apoptosis in chondrocytes, we could find no correlation of NO release and cell death in our study, possibly due to the late induction of NO. Furthermore, the explicit role of $\mathrm{NO}$ in cell death and apoptosis is still a matter of discussion (26).

The difference in the mediator composition caused by additional IL- $1 \beta$ stimulation of impacted cartilage explants was not reflected in differences in cell viability, however, differential effects of a p38MAPK inhibition could be detected. We observed that SB203580 tended to reduce cell death in impacted and IL-1 $\beta$-stimulated explants. The protective trend was accompanied by a further elevation of IL-6 and NO levels, whereas the PGD2 level was not altered. The increased levels of IL- 6 released under IL- $1 \beta$ stimulation may have protective effects, as IL-6 plays a major role in tendon reparative processes (27). IL- 6 is able to activate the Jak/STAT3 pathway implicated in cell proliferation and survival (28).

In contrast, p38MAPK inhibition could not increase the number of viable cells in impacted explants without IL-1 $\beta$ stimulation, but resulted in a reduced IL- 6 and an increased PGD2 release. The latter effect might be explained by a possible COX1-dependent PGD2 synthesis, primarily described in macrophages (29) and a COX1 induction through mechanical loading, observed in osteoblasts (30). Assuming comparable mechanisms in chondrocytes, an imbalance in the arachidonic acid cascade by $\mathrm{p} 38$ inhibition could result in enhanced metabolism via COX1 and a subsequent increased PGD2 release. As PGD2 is considered to play a role in chondrocyte apoptosis (23), SB203580-induced PGD2 increase may reduce cell viability and level out other protective effects of the inhibitor. A comparable effect of p38MAPK inhibition was also described for mechanically-induced apoptosis of intervertebral disc chondrocytes (31).

An explanation for the different effects of p38MAPK inhibition with and without concomitant IL- $1 \beta$ stimulation can only be assumed. Trauma as well as IL-1 $\beta$ may induce destructive and protective processes via diverse signaling pathways. The balance of these processes may be displaced in different directions by the inhibition of an involved pathway.

In contrast to our findings, other studies described a significant reduction of injury-related chondrocyte death by p38 inhibition in cartilage trauma models without concomitant IL-1 $\beta$ stimulation $(13,19)$. The discrepancies may be due to differences in the experimental designs. One study (19) applied the p38 inhibitor 90 min prior impaction in order to abolish immediate p38 signaling and both studies used an inhibitor concentration higher than that in our study (10 and 20-fold, respectively), that might result in inhibition of additional kinases, such as JNK (32). We opted to apply the inhibitor post-trauma in order to remain within a possible therapeutic time frame and selected an inhibitor concentration that could ensure specificity. The question of optimal selective and effective inhibitor concentrations under tissue culture conditions remains open. Furthermore, the use of human osteoarthritic cartilage in our study may account for speciesand age-dependent differences or may reflect OA-associated changes of the cartilage such as bio-distribution and/or preexisting synthesis of prostaglandins and other mediators.

In addition, not only negative but also protective signals could be mediated by p38, and could be abolished by p38 inhibition. Our results clearly indicate that one has to be aware of the highly complex interactions of the different players activating and modulating signaling pathways.

In summary, we showed that a single impact induces an increased PGD2 release and other trauma-typical effects in human early-stage osteoarthritic cartilage explants, showing that aged osteoarthritic chondrocytes are still susceptible for mechanical injury. The pro-inflammatory cytokine IL-1 $\beta$ had no additive effect on trauma-induced cell loss in contrast to the described synergistic effect of IL-1 and trauma on proteoglycan loss in cartilage (11). A cocktail of mediators was induced by trauma alone and by trauma with IL- $1 \beta$ stimulation, differing in composition and time-response. In tendency, p38MAPK inhibition diminished trauma-induced chondrocyte death in an inflammatory environment, suggesting a role for p38MAPK signaling in cell viability after impact, as described by others $(13,19)$.

\section{Acknowledgements}

The authors would like to thank Brunhilde Amann for excellent technical assistance. This study was supported by the German Research Council (DFG, Grant KFO 200).

\section{References}

1. Furman BD, Olson SA and Guilak F: The development of posttraumatic arthritis after articular fracture. J Orthop Trauma 20: 719-725, 2006. 
2. Szczodry M, Coyle CH, Kramer SJ, Smolinski P and Chu CR Progressive chondrocyte death after impact injury indicates a need for chondroprotective therapy. Am J Sports Med 37: 2318-2322, 2009.

3. Irie $\mathrm{K}$, Uchiyama $\mathrm{E}$ and Iwaso $\mathrm{H}$ : Intraarticular inflammatory cytokines in acute anterior cruciate ligament injured knee. Knee 10: 93-96, 2003.

4. Phillips DM and Haut RC: The use of a non-ionic surfactant (P188) to save chondrocytes from necrosis following impact loading of chondral explants. J Orthop Res 22: 1135-1142, 2004

5. D'Lima DD, Hashimoto S, Chen PC, Colwell CW and Lotz MK Human chondrocyte apoptosis in response to mechanical injury. Osteoarthritis Cartilage 9: 712-719, 2001.

6. Kurz B, Lemke A, Kehn M, et al: Influence of tissue maturation and antioxidants on the apoptotic response of articular cartilage after injurious compression. Arthritis Rheum 50: 123-130, 2004.

7. DiMicco MA, Patwari P, Siparsky PN, et al: Mechanisms and kinetics of glycosaminoglycan release following in vitro cartilage injury. Arthritis Rheum 50: 840-848, 2004.

8. Stevens AL, Wishnok JS, White FM, Grodzinsky AJ and Tannenbaum SR: Mechanical injury and cytokines cause loss of cartilage integrity and upregulate proteins associated with catabolism, immunity, inflammation, and repair. Mol Cell Proteomics 8: 1475-1489, 2009.

9. Goldring MB and Marcu KB: Cartilage homeostasis in health and rheumatic diseases. Arthritis Res Ther 11: 224, 2009.

10. Radons J, Bosserhoff AK, Graessel S, Falk W and Schubert TE: p38MAPK mediates IL-1-induced down-regulation of aggrecan gene expression in human chondrocytes. Int J Mol Med 17: 661-668, 2006.

11. Patwari P, Cook MN, DiMicco MA, et al: Proteoglycan degradation after injurious compression of bovine and human articular cartilage in vitro: interaction with exogenous cytokines. Arthritis Rheum 48: 1292-1301, 2003.

12. Sui Y, Lee JH, DiMicco MA, et al: Mechanical injury potentiates proteoglycan catabolism induced by interleukin- 6 with soluble interleukin- 6 receptor and tumor necrosis factor alpha in immature bovine and adult human articular cartilage. Arthritis Rheum 60: 2985-2996, 2009

13. Bajaj S, Shoemaker T, Hakimiyan AA, et al: Protective effect of P188 in the model of acute trauma to human ankle cartilage: the mechanism of action. J Orthop Trauma 24: 571-576, 2010.

14. Huser CAM, Peacock M and Davies ME: Inhibition of caspase-9 reduces chondrocyte apoptosis and proteoglycan loss following mechanical trauma. Osteoarthritis Cartilage 14: 1002-1010, 2006.

15. Goodwin W, McCabe D, Sauter E, et al: Rotenone prevents impact-induced chondrocyte death. J Orthop Res 28: 1057-1063, 2010.

16. Jeffrey JE and Aspden RM: Cyclooxygenase inhibition lowers prostaglandin E2 release from articular cartilage and reduces apoptosis but not proteoglycan degradation following an impact load in vitro. Arthritis Res Ther 9: R129, 2007.

17. Joos H, Albrecht W, Laufer S and Brenner RE: Differential effects of p38MAP kinase inhibitors on the expression of inflammation-associated genes in primary, interleukin-1betastimulated human chondrocytes. Br J Pharmacol 160: 1252-1262, 2010.
18. Brown KK, Heitmeyer SA, Hookfin EB, et al: P38 MAP kinase inhibitors as potential therapeutics for the treatment of joint degeneration and pain associated with osteoarthritis. J Inflamm (Lond) 5: 22, 2008.

19. Ding L, Heying E, Nicholson N, et al: Mechanical impact induces cartilage degradation via mitogen activated protein kinases. Osteoarthritis Cartilage 18: 1509-1517, 2010.

20. Takebe K, Nishiyama T, Hayashi S, et al: Regulation of p38 MAPK phosphorylation inhibits chondrocyte apoptosis in response to heat stress or mechanical stress. Int J Mol Med 27: 329-335, 2011

21. D'Lima DD, Hashimoto S, Chen PC, Colwell CW Jr and Lotz K: Impact of mechanical trauma on matrix and cells. Clin Orthop Relat Res 391 (Suppl.): S90-S99, 2001.

22. Green DM, Noble PC, Ahuero JS and Birdsall HH: Cellular events leading to chondrocyte death after cartilage impact injury. Arthritis Rheum 54: 1509-1517, 2006.

23. Zhu F, Wang P, Kontrogianni-Konstantopoulos A and Konstantopoulos K: Prostaglandin (PG)D(2) and 15-deoxyDelta(12,14)-PGJ(2), but not PGE(2), mediate shear-induced chondrocyte apoptosis via protein kinase A-dependent regulation of polo-like kinases. Cell Death Differ 17: 1325-1334, 2010.

24. Zayed N, Li X, Chabane N, et al: Increased expression of lipocalin-type prostaglandin D2 synthase in osteoarthritic cartilage. Arthritis Res Ther 10: R146, 2008.

25. Fermor B, Weinberg JB, Pisetsky DS, Misukonis MA, Banes AJ and Guilak F: The effects of static and intermittent compression on nitric oxide production in articular cartilage explants. J Orthop Res 19: 729-737, 2001.

26. Abramson SB: Osteoarthritis and nitric oxide. Osteoarthritis Cartilage 16 (Suppl. 2): S15-S20, 2008.

27. Lin TW, Cardenas L, Glaser DL and Soslowsky LJ: Tendon healing in interleukin- 4 and interleukin- 6 knockout mice. J Biomech 39: 61-69, 2006.

28. Nishimoto $\mathrm{N}$ and Kishimoto $\mathrm{T}$ : Interleukin 6: from bench to bedside. Nat Clin Pract Rheumatol 2: 619-626, 2006.

29. Matsumoto H, Naraba H, Murakami M, et al: Concordant induction of prostaglandin E2 synthase with cyclooxygenase-2 leads to preferred production of prostaglandin E2 over thromboxane and prostaglandin D2 in lipopolysaccharide-stimulated rat peritoneal macrophages. Biochem Biophys Res Commun 230: $110-114,1997$.

30. Siddhivarn C, Banes A, Champagne C, Riche EL, Weerapradist W and Offenbacher S: Prostaglandin D2 pathway and peroxisome proliferator-activated receptor gamma-1 expression are induced by mechanical loading in an osteoblastic cell line. J Periodontal Res 41: 92-100, 2006.

31. Ariga K, Yonenobu K, Nakase T, et al: Mechanical stressinduced apoptosis of endplate chondrocytes in organ-cultured mouse intervertebral discs: an ex vivo study. Spine 28: 1528-1533, 2003.

32. Karaman MW, Herrgard S, Treiber DK, et al: A quantitative analysis of kinase inhibitor selectivity. Nat Biotechnol 26: $127-132,2008$ 